\title{
ENGENHARIA DIDÁTICA COMO INSTRUMENTO METODOLÓGICO NO ESTUDO E NO ENSINO DA SEQUÊNCIA DE JACOBSTHAL
}

\author{
Thamires Silva Aquino de Souza* \\ Francisco Regis Vieira Alves ${ }^{* *}$
}

\begin{abstract}
Resumo: O trabalho em tela tem por finalidade tecer discussões sobre alguns modelos de generalização e extensão da Sequência de Jacobsthal. Assim, em consonância com as orientações da Engenharia Didática como metodologia de pesquisa e em complementariedade com a Teoria das Situações Didáticas, que permite uma análise minuciosa dos fenômenos relativos ao ensino e aprendizagem de Sequência de Jacobsthal, descreveremos os elementos principais que constituem as duas fases iniciais de uma Engenharia Didática, a saber análise preliminar e análise a priori, ressaltando a concepção de situações didáticas e os possíveis comportamentos e resoluções dos alunos diante das duas situações problemas, organizadas com fins à experimentação.
\end{abstract}

Palavras-chave: Engenharia Didática. Teoria das Situações Didáticas. Sequência de Jacobsthal.

\section{Introdução}

Indubitavelmente, a sequência de Fibonacci configura-se como uma das mais importantes e conhecidas no mundo inteiro. Objeto de estudo ao longo de vários anos, até hoje resguarda um caráter relevante e enigmático. Tal sequência originou-se de um problema relativo à reprodução infinita de pares de coelhos.

Em contrapartida, observamos nos compêndios especializados em História da Matemática uma inclinação a evidenciar os pressupostos do Cálculo Diferencial e Infinitesimal, discutindo a historicidade dos conceitos matemáticos que lhe são intrínsecos, bem como o seu desenvolvimento desde sua gênese até a contemporaneidade (ALVES, 2018).

Desse modo, a abordagem dada à sequência de Fibonacci é mostrada de maneira sucinta e trivial, podendo levar à um entendimento raso e deslocado, principalmente no que diz respeito ao seu processo diacrônico. E, além disso, não proporcionam ao leitor uma compreensão sobre o estado atual e evolutivo, a partir de uma perspectiva contigua, evolutiva e prolongada.

\footnotetext{
* Docente do Instituto Federal de Educação, Ciência e Tecnologia - IFCE. Discente do Mestrado em Ensino de Ciências e Matemática - PGECM/IFCE.

*** Docente do Mestrado em Ensino de Ciências e Matemática - PGECM/IFCE. Docente do Mestrado em Ensino de Ciências e Matemática - ENCIMA/UFC. Docente do Mestrado em Educação Profissional e Tecnológica PROEPT/IFCE.
} 


\section{\#tear}

Nesse contexto, evidenciamos o surgimento de diversas sequências numéricas recursivas, consequência da mobilização de matemáticos do mundo inteiro que tinham como escopo em fornecer novos modelos generalizados, como por exemplo a sequência de Jacobsthal, que é definida pela seguinte relação de recorrência $J_{n}=J_{n-1}+2 J_{n-2}, n \geq 2$, tais que as condições iniciais são $J_{0}=0, J_{1}=1$ que descreve o seguinte conjunto numérico: $\left\{J_{n}\right\}_{n \in I N}=\{0,1,1,3,5,11,21,43,85,171, \ldots\}$ e possui propriedades que se relacionam com outros conceitos matemáticos.

Tal sequência recebe esse nome como referência ao matemático Ernest Erich Jacobsthal (1882-1965), especialista em Teoria dos Números e ex-aluno de Ferdinand G. Frobenius, que foi um dos primeiros a estudar/ definir os polinômios de Fibonacci.

No Brasil são escassos os trabalhos relativos à Sequência de Jacobsthal. Encontramos alguns que apresentam extensões e interpretações combinatórias para os números de Jacobsthal via ladrilhamentos (Craveiro, 2004; Spreafico, 2014; Silva, 2014). Estes, porém, não apresentam propriedades iniciais sobre a sequência de Jacobsthal e seus diversos tipos de representação. Em Alves (2017), encontramos propriedades relativas à sequência generalizada de Jacobsthal, bem como a exploração de conceitos matriciais relativos à temática que se mostram ainda pouco discutidas em nosso país.

Destarte, adotamos como objetivo a descrição de um tratamento para o tema "Sequência de Jacobsthal", tendo como base alguns elementos de uma Engenharia Didática clássica ou Engenharia Didática de $1^{a}$ geração, como forma de sistematizar e organizar a pesquisa, tendo em vista a etapa de concepção, a experimentação, a descrição de situações dialéticas em seus tempos ou fases previstas de ação, formulação, validação e institucionalização, propostas pela Teoria Das Situações Didáticas.

\section{A fórmula de Binet como modelo de generalização da sequência de Jacobsthal}

Conforme mencionado anteriormente, a sequência de Jacobsthal é dada pela lei de recorrência $J_{n}=J_{n-1}+2 J_{n-2}, n \geq 2$, tais que as condições iniciais são $J_{0}=0, J_{1}=1$ que descreve o seguinte conjunto numérico: $\left\{J_{n}\right\}_{n \in I N}:\{0,1,1,3,5,11,21,43,85,171, \ldots\}$.

A respeito dessa temática, destacamos o próprio trabalho de Ernest Jacobsthal (18821965), intitulado Fibonaccische Polynome und Kreisteilungsgleichungen, em que o mesmo apresenta propriedades relativas aos polinômios de Fibonacci e equações circulares. Na figura 


\section{\#tear}

a seguir, apresentamos parte importante de seu trabalho que envolve uma forte abordagem de pesquisa em termo de sequências recorrentes que enfatizaram representações matriciais.

Figura 1 - Propriedades das sequências numéricas estudadas por Jacobsthal werte $x$ eintreten. Um diese zu charakterisieren, bemerke ich, daB die Substitution (5) sich auch in der Form

$$
z_{n}=\left(\begin{array}{ll}
1 & x \\
1 & 0
\end{array}\right)^{n} z_{0}
$$

$$
(n=0,1,2, \ldots)
$$

schreiben läBt Diese Gleichung sieht nach Ausrechnung der Potenz $\left(\begin{array}{l}1 \\ 1\end{array}\right)^{n}$ so aus:

$$
z_{n}=\left(\begin{array}{ll}
f_{n} & x f_{n-1} \\
f_{n-1} & x f_{n-2}
\end{array}\right) z_{0},
$$

Hierin bedeutet $f_{n}=f_{n}(x)$ cine ganze ganzzahlige Funktion von $x$, die dem Rekursionsgesetze

$$
f_{x+1}=f_{n}+x f_{n-1}, \quad f_{-1}=0, f_{0}=1 \text {, }
$$$$
(n=0,1,2, \ldots)
$$

genügt. Bekanntlich sind die Zahlen $f_{n}(1)$ die sogenannten Fibonaccischen Zahlen. Man nennt daher die Polynome $f_{n}(x)$ die Fibonaccischen Polynome.

Fonte: Jacobsthal (1919-1920)

Com base na figura anterior, destacamos a equação de recorrência $f_{n+1}=f_{n}+x \cdot f_{n-1}$. Fundamentados na análise da mesma, observamos que para $x=1$ obtemos a sequência de Fibonacci. Para $x=2$ resulta a sequência de Jacobsthal mencionada anteriormente. Por outro lado, podemos extrair também propriedades relativas às matrizes de ordem 2.

Não obstante, notamos que, para a obtenção de um número qualquer de Jacobsthal, é necessário conhecer os seus dois elementos anteriores e, em seguida, utilizar a relação de recorrência. A título de exemplo, se desejarmos obter o número $J_{70}$ é necessário estabelecer os elementos $J_{69}$ e $J_{68}$ e prosseguir com a relação de recorrência.

Um tópico que merece investigação é sobre a possibilidade de obter os termos dessa sequência sem a necessidade de utilizar a relação de recorrência mencionada anteriormente. Para tal, adotamos o emprego da fórmula de Binet. Segundo Horadan (1996), essa fórmula é obtida considerando-se $\alpha=2$ e $\beta=-1$, que são raízes da equação característica $x^{2}-x-2=0$

Entre as muitas propriedades existentes, podemos mencionar: $\alpha^{2}=\alpha+2 ; \alpha+\beta=1 ; \alpha \cdot \beta=-2 ; \alpha-\beta=3 ; \beta^{2}=\beta+2 ; \alpha^{2}-\beta^{2}=3$.

Prosseguindo, temos que as raízes desta equação devem satisfazer a $\alpha^{2}=\alpha+2$ e $\beta^{2}=\beta+2$. Multiplicando a primeira dessas relações $\alpha^{n}$, e a segunda por $\beta^{n}$, obtemos 
(i) $\alpha^{n+2}=\alpha^{n+1}+2 \alpha^{n}$ e $\beta^{n+2}=\beta^{n+1}+2 \cdot \beta^{n}$ (ii). Subtraindo a segunda equação da primeira, e dividindo ambas por $\alpha-\beta$, chegamos a: $\frac{\alpha^{n+2}-\beta^{n+2}}{\alpha-\beta}=\frac{\alpha^{n+1}-\beta^{n+1}}{\alpha-\beta}+2 \cdot\left(\frac{\alpha^{n}-\beta^{n}}{\alpha-\beta}\right)$. Se colocarmos $H_{n}=\frac{\alpha^{n}-\beta^{n}}{\alpha-\beta}$, temos: $H_{n+2}=H_{n+1}+2 H_{n}$. Temos $\alpha+\beta=1, \alpha-\beta=3, \alpha \cdot \beta=-2$, consequentemente obtemos:

$$
\begin{aligned}
& H_{1}=\frac{\alpha-\beta}{\alpha-\beta}=1, H_{2}=\frac{\alpha^{2}-\beta^{2}}{\alpha-\beta}=\alpha+\beta=1, H_{3}=\frac{\alpha^{3}-\beta^{3}}{\alpha-\beta}=\frac{(\alpha-\beta)\left(\alpha^{2}+\alpha \beta+\beta^{2}\right)}{\alpha-\beta}=3 \\
& \text { e } H_{4}=\frac{\left(\alpha^{2}-\beta^{2}\right) \cdot\left(\alpha^{2}+\beta^{2}\right)}{\alpha-\beta}=(\alpha+\beta) \cdot\left(\alpha^{2}+\beta^{2}\right)=5 . \text { Com isto, os resultados } H_{1}, H_{2}, H_{3}, \cdots,
\end{aligned}
$$
representam a sequência de Jacobsthal, com $J_{n}=\frac{\alpha^{n}-\beta^{n}}{\alpha-\beta}$, onde $n \geq 1$

Isso posto, observamos que a fórmula de Binet permite a obtenção dos termos inteiros da sequência de Jacobsthal, sem precisarmos utilizar os conceitos de recursividade, atuando assim, como um modelo de generalização da sequência de Jacobsthal. A seguir, podemos formalizar um modelo de Generalização para essa sequência por intermédio da fórmula de Binet com a apresentação do teorema a seguir:

Teorema 1: Dado um número $n \geq 1$, então $J_{n}=\frac{\alpha^{n}-\beta^{n}}{3}=\frac{1}{3}\left(2^{n}-(-1)^{n}\right)$, onde os números $\alpha=2, \beta=-1$ correspondem às duas raízes da equação $x^{2}-x-2=0$.

Demonstração:

A demonstração imediata segue por Indução Matemática.

Para $n=1$, temos $J_{1}=\frac{2^{1}-(-1)^{1}}{3}=\frac{2+1}{3}=1$

Supomos a validade para $n=1, \cdots, \mathrm{k}$. Vamos mostrar que é válida para $k+1$.

$$
\begin{aligned}
& J_{k+1}=J_{k}+2 J_{k-1}=\frac{\alpha^{k}-\beta^{k}}{\alpha-\beta}+2\left(\frac{\alpha^{k-1}-\beta^{k-1}}{\alpha-\beta}\right)=\frac{\alpha^{k}-\beta^{k}+2 \alpha^{k-1}-2 \beta^{k-1}}{\alpha-\beta}=\frac{\alpha^{k}\left(1+2 \alpha^{-1}\right)-\beta^{k}\left(1+2 \beta^{-1}\right)}{3}= \\
& =\frac{\alpha^{k} \cdot \alpha-\beta^{k} \cdot \beta}{3}=\frac{2^{k} \cdot 2-(-1)^{k} \cdot(-1)}{3}=\frac{2^{k+1}-(-1)^{k+1}}{3} \text { (c.q.d) }
\end{aligned}
$$

Ainda no que se refere à formula de Binet, apresentaremos algumas considerações sobre esse assunto em seu modelo matricial.

As investigações de Koken \& Bozkurt (2008) apresentam uma discussão que relaciona os números de Jacobsthal com matrizes. Iniciaremos nosso estudo apresentando a 
matriz que os autores caracterizam como $F=\left[\begin{array}{ll}1 & 2 \\ 1 & 0\end{array}\right]$. Seguindo um raciocínio comum às pesquisas mencionadas, obtemos alguns casos do produto entre matrizes: $F^{2}=F \cdot F=\left[\begin{array}{ll}1 & 2 \\ 1 & 0\end{array}\right] \cdot\left[\begin{array}{ll}1 & 2 \\ 1 & 0\end{array}\right]=\left[\begin{array}{ll}3 & 2 \\ 1 & 2\end{array}\right], F^{3}=F^{2} \cdot F=\left[\begin{array}{ll}3 & 2 \\ 1 & 2\end{array}\right] \cdot\left[\begin{array}{ll}1 & 2 \\ 1 & 0\end{array}\right]=\left[\begin{array}{ll}5 & 6 \\ 3 & 2\end{array}\right]$ e $F^{4}=F^{3} \cdot F=\left[\begin{array}{ll}5 & 6 \\ 3 & 2\end{array}\right] \cdot\left[\begin{array}{ll}1 & 2 \\ 1 & 0\end{array}\right]=\left[\begin{array}{cc}11 & 10 \\ 5 & 6\end{array}\right]$. De acordo com os casos levantados, podemos conjecturar o seguinte resultado: $F^{n}=\left[\begin{array}{cc}J_{n+1} & 2 J_{n} \\ J_{n} & 2 J_{n-1}\end{array}\right]$. Assim, enunciamos o seguinte teorema:

Teorema 2: Para um dado $n \in \mathbb{N}$ a enésima potência da matriz $F$ é dada por: $F^{n}=\left[\begin{array}{cc}J_{n+1} & 2 J_{n} \\ J_{n} & 2 J_{n-1}\end{array}\right]$, sendo $J_{n+1}, J_{n}$ e $J_{n-1}$ números de Jacobsthal.

Demostração: Faremos a demonstração do teorema anterior utilizando o princípio da indução matemática. Assim, para $n=1$, temos: $F^{1}=\left[\begin{array}{cc}J_{2} & 2 J_{1} \\ J_{1} & 2 J_{0}\end{array}\right]$. Assim, suponhamos a validade para $n=k$, temos $F^{k}=\left[\begin{array}{cc}J_{k+1} & 2 J_{k} \\ J_{k} & 2 J_{k-1}\end{array}\right]$. Vamos mostrar a validade para $n=k+1$. Então, $F^{k+1}=F^{k} \cdot F=\left[\begin{array}{cc}J_{k+1} & 2 J_{k} \\ J_{k} & 2 J_{k-1}\end{array}\right] \cdot\left[\begin{array}{cc}1 & 2 \\ 1 & 0\end{array}\right]=\left[\begin{array}{cc}J_{k+1}+2 J_{k} & 2 J_{k+1} \\ J_{k}+2 J_{k-1} & 2 J_{k}\end{array}\right]=\left[\begin{array}{cc}J_{k+2} & 2 J_{k+1} \\ J_{k+1} & 2 J_{k}\end{array}\right]$ (c.q.d $)$

Tendo em vista que matrizes e determinantes são conteúdos que estão intimamente relacionados, apresentamos o próximo teorema que nos informa o determinante da matriz $F^{n}$. Teorema 3: Para um dado inteiro n, temos $\operatorname{det}\left(F^{n}\right)=(-2)^{n}$.

Demonstração: dos estudos relativos aos determinantes sabemos que $\operatorname{det}\left(F^{n}\right)=(\operatorname{det} F)^{n}$. Temos que $\operatorname{det} F=\left|\begin{array}{ll}1 & 2 \\ 1 & 0\end{array}\right|=-2$, concluímos que: $\operatorname{det}(F)^{n}=(-2)^{n}$.

Com base no teorema anterior, vamos demonstrar uma identidade que é atribuída ao matemático italiano Giovanni Domenico Cassini (1625-1712), que propôs a seguinte relação para a Sequência de Fibonacci $f_{n-1} f_{n+1}-f_{n}^{2}=(-1)^{n}$ (KOSHY,2011). Observando a identidade anterior e tendo como apoio os trabalhos de Koken \& Bozkurt (2008), apresentaremos um modelo de extensão da identidade de Cassini para a Sequência de Jacobsthal. 
Teorema 4: Considerando a matriz $F^{n}=\left[\begin{array}{cc}J_{n+1} & 2 J_{n} \\ J_{n} & 2 \cdot J_{n-1}\end{array}\right]$ vale que: $J_{n-1} J_{n+1}-J_{n}^{2}=(-1)^{n-1} \cdot 2^{n-1}$ Demonstração: Sabendo que $F^{n}=\left[\begin{array}{cc}J_{n+1} & 2 J_{n} \\ J_{n} & 2 J_{n-1}\end{array}\right]$ e det $F=(-2)$. Dessa forma, teremos: $(-2)^{n}=\operatorname{det} F^{n}=\operatorname{det}\left[\begin{array}{cc}J_{n+1} & 2 \cdot J_{n} \\ J_{n} & 2 \cdot J_{n-1}\end{array}\right]=2 \cdot J_{n-1} \cdot J_{n+1}-2 \cdot J_{n} \cdot J_{n}$ e, dessa forma, segue que $J_{n-1} J_{n+1}-J_{n}^{2}=(-2)^{n-1}=(-1)^{n-1} \cdot 2^{n-1}($ c.q.d $)$

\section{Alguns pressupostos da engenharia didática}

Sobre o campo epistêmico de estudos nominado Engenharia Didática, percebemos uma série de influências que culminaram na delimitação dessa área de estudos. A seguir apresentaremos as principais contribuições para constituição da ED.

Podemos destacar inicialmente a influência de um viés piagetiano, no que diz respeito a objetivar situações de ensino pautadas na análise minuciosa do indivíduo em situações de aprendizagem. Por outro lado, destacamos os traços do pensamento de Bachelard (1996), no que diz respeito à descrição de obstáculos epistemológicos. A noção de obstáculos epistemológicos foi concebida originalmente para a Física, mas vale salientar que sua ideia pode ser expandida para outras áreas do conhecimento.

Assim, de maneira análoga ao caso de Bachelard, Brousseau adaptou esse conceito para a matemática e aponta o expediente inescapável dos obstáculos, procedente da interação dialética entre "conhecimento" e "meio". De fato, assegura que:

Um conhecimento, como obstáculo, é sempre fruto de uma interação de um aluno com o meio e, mais precisamente com uma situação que torna o conhecimento interessante [...] todavia, tais concepções são comandadas por intermédio das condições de interação. (BROUSSEAU, 1976, p.107)

Nessa perspectiva, apresentamos outra corrente de grande importância para a Engenharia Didática. Estamos nos referindo à Didática da Matemática, que se desenvolveu principalmente na França do final dos anos 60 com o surgimento dos Institutos de Investigação acerca do Ensino das Matemáticas (IREM). A Didática da Matemática tem como marco a reforma da matemática moderna, bem como o êxito das teorias piagetianas sobre a psicologia do desenvolvimento. Nesse sentido, evidenciamos a Teoria das Situações Didáticas, descrita por Brousseau (1986), que estuda as formas de elaboração e apresentação do saber escolar. 


\section{\#tear}

Nesse sentido, Artigue (1995), evidencia que a Teoria das Situações Didáticas, passou por alguns entraves metodológicos e assim culminou no desenvolvimento, em oposição aos paradigmas clássicos de experimentação em sala de aula, de uma metodologia específica: A Engenharia Didática.

Assim, Artigue (1995) ao apresentar o termo Engenharia Didática faz uma analogia entre a forma de trabalho do professor e o trabalho de um engenheiro que para realizar um projeto específico, baseia-se em um conhecimento científico e aceita submeter-se à um controle do tipo científico. Ao mesmo tempo, é obrigado a trabalhar objetos bem mais complexos do que os objetos depurados da ciência e, portanto, enfrentar, com todos os meios de que dispõe, problemas que a ciência não quer ou não pode assumir.

Por outro lado, ainda conforme Artigue (1995) a Engenharia Didática vista como metodologia de pesquisa é inicialmente caracterizada por um esquema experimental baseado em "realizações didáticas" em sala de aula. Esse esquema consiste na concepção, realização, observação e análise de sequências de ensino.

Nesse escopo, a engenharia didática apresenta dois níveis de pesquisa microengenharia e macroengenharia. A primeira diz respeito à uma visão mais restrita na medida em que se interessa pelas relações e fenômenos que ocorrem no interior da própria sala de aula e "são mais fáceis de desenvolver na prática" (ARTIGUE, 1995, p.36). Já a segunda, macroengenharia, diz respeito às dificuldades de cunho metodológico e/ou institucional e envolvem um interesse de identificação de condicionantes oriundos do funcionamento da escola ou de uma instituição (ALVES, 2016).

A pesquisa que ora apresentamos trata-se de uma microengenharia que busca desenvolver uma Engenharia Didática no ensino de História da Matemática relativo ao conteúdo sequência de Jacobsthal no campo dos inteiros. Além disso, apresentamos também a sistematização de variáveis macrodidáticas que dizem respeito à organização global e as variáveis microdidáticas, que se relacionam à um estágio específico da engenharia, sobretudo, à fase da experimentação.

Outrossim, a Engenharia Didática que visamos se apoia em sua fase de experimentação, na metodologia de ensino nomeada Teoria das Situações Didáticas - TSD, conforme foi mencionado anteriormente. Essa teoria foi desenvolvida especificamente para o ensino de matemática e pretende produzir um modelo de interação entre o aluno, o saber e o ambiente de aprendizagem no qual o conhecimento deverá se constituir (ALMOULOUD, 2007). 
Com fins a organizar o processo de aprendizagem, a Teoria das Situações Didáticas analisa e desmembra esse processo em quatro etapas dissemelhantes, nas quais o educando tem relações distintas com o saber. São as fases de: ação, formulação, validação e institucionalização. É essencial, destacar o caráter de complementariedade da ED com a TSD, tendo em vista que a Teoria das Situações Didáticas servirá de amparo para Engenharia Didática.

Ademais, no que tange às etapas de investigação de uma Engenharia Didática, observamos: as análises preliminares; análises a priori; experimentação; análise a posteriori e validação. Nesta investigação, nossos estudos serão balizados pelas duas fases iniciais previstos por Artigue (1995), a saber análises preliminares e análises a priori.

\section{Análises preliminares e o estado da arte}

Segundo os pressupostos da Engenharia Didática, como forma de sistematizar a análise preliminar, é recomendável proceder a uma descrição das principais dimensões que definem o fenômeno a ser analisado e que se relacionam com o sistema de ensino, quais sejam a didática, a epistemológica e a cognitiva. Cada uma dessas perspectivas atua na constituição do objeto de estudo (PAIS, 2015).

Desse modo, no que concerne à dimensão didática, analisamos a abordagem dada à sequência de Jacobsthal, por meio de uma pesquisa de cunho bibliográfico, buscando elucidar as definições e propriedades referentes a esse objeto que foram apresentadas em artigos científicos tais como: Horadam (1996), Koken \& Bozkurt (2008) e alguns mais recentes como: Gnanam \& Anitha (2016) e Alves (2017). Essa análise nos permite traçar caminhos para a realização da transposição didática, ou seja, transformar o conteúdo científico em um conteúdo que possa ser ensinado em sala de aula.

Sobre a dimensão epistemológica, foram considerados aspectos tais como a origem e o progresso dos conceitos matemáticos relativos à sequência de Jacobsthal. Além disso, divisamos as relações desse objeto com outros conceitos matemáticos. Na figura a seguir, apresentamos um encadeamento de temas matemáticos que podemos associar à sequência de Jacobsthal e sugerir um amplo repertório de relações matemáticas conceituais imprescindíveis tanto para os estudantes como, também, a partir de uma perspectiva de ensino de Matemática. 
Figura 2 - Associação da sequência de Jacobsthal à outros conteúdos matemáticos

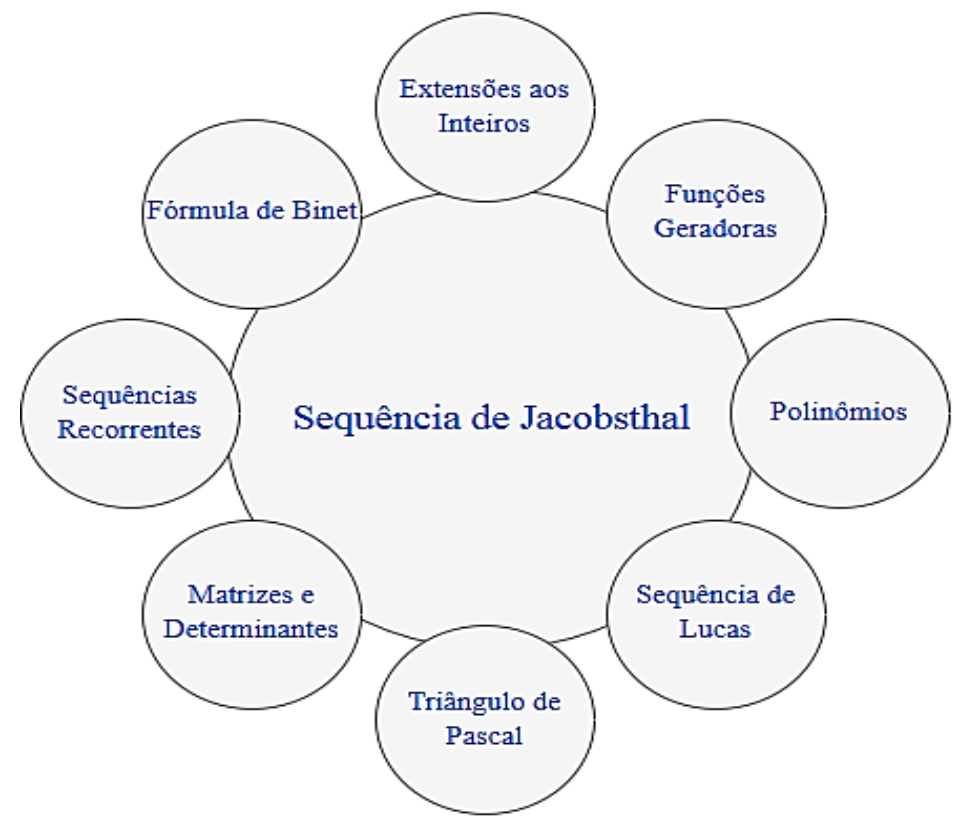

Fonte: Elaboração dos autores

Com base na figura acima percebemos as possíveis formas de relacionar o objeto matemático em questão com outros conteúdos estudados na academia. É perceptível que podemos fazer analogias entre a sequência de Jacobsthal e outros temas matemáticos que vão desde os mais simples, como os estudados no ensino médio, até os mais abstratos que são contemplados apenas no ensino superior. Desse modo, confirmamos sua evolução tanto no âmbito epistêmico quanto no que concerne ao desenvolvimento matemático gradativo.

No que se refere à dimensão cognitiva, pretende-se propor situações didáticas de modo que o aluno possa discernir e manifestar a intuição, conduzindo-o ao desenvolvimento de um raciocínio lógico-dedutivo e desse modo mobilizar o conhecimento deste tópico.

Ainda no que diz respeito às análises preliminares da Engenharia Didática, Pommer (2013, p.23) afirma que "é feita uma revisão bibliográfica envolvendo as condições e contextos presentes nos vários níveis de produção didática”. Além disso, Almouloud e Silva (2012), apontam que nesse estágio realiza-se considerações sobre o quadro teórico geral.

Nessa perspectiva, o estado da arte assemelha-se à análise que é feita na primeira fase da Engenharia Didática. Segundo Ferreira (2002), o Estado da Arte é uma pesquisa de cunho bibliográfico com objetivo de mapear e discutir determinada produção acadêmica. Além disso, trata-se de "uma metodologia de caráter inventariante e descritivo da produção acadêmica e científica sobre o tema que busca investigar”. (FERREIRA,2012, p. 258). 
Para a realização do Estado da Arte foi definido o período de corte de 2013 a 2018 (5 anos). Selecionamos artigos de matemática pura em periódicos internacionais, tendo em vista a escassez de trabalhos sobre a temática no Brasil. Para a identificação dos artigos foi realizada a leitura do título, resumo e palavras-chave que abrangessem os seguintes termos: Jacobsthal numbers; Binet's formula; Negative Jacobsthal Numbers; Generalized Jacobsthal sequence e termos associados à sequência de Jacobsthal.

Após a pesquisa foram selecionados cinco trabalhos em cinco periódicos diferentes. De maneira geral, os artigos versam sobre pesquisas mais recentes sobre a sequência de Jacobsthal. A seguir, apresentamos uma breve análise de cada um dos artigos.

O trabalho de Cook e Bacon (2013) tem como objetivo propor algumas identidades adicionais para os números de Jacobsthal e de Jacobsthal- Lucas. Além disso, são apresentadas relações análogas para os números que surgem de algumas relações de recorrência de Jacobsthal de ordem superior. Ao longo do escrito, são explorados os desenvolvimentos matemáticos dessas propriedades. Em conclusão, os autores propõem futuras investigações, quando afirmam que outras identidades podem ser obtidas a partir das que foram mencionadas na publicação.

Na produção de Campos et. al (2014), é apresentada a sequência de k-JacobsthalLucas. Esse tipo de sequência apresenta uma espécie de generalização que foi proposta por Horadam (1998). Assim, os autores estabelecem algumas adaptações da fórmula de Binet e as identidades de Catalan e d'Ocagne para os números de k-Jacobsthal- Lucas. O trabalho é finalizado com a apresentação das funções geradoras para essa sequência.

Catarino et. al (2015) sugere uma abordagem para novas famílias de sequências do tipo $J_{n}^{(k)}$ que generalizam os números Jacobsthal e Jacobsthal-Lucas. Os autores apresentam inicialmente as propriedades básicas e gradativamente realizam o desenvolvimento algébrico dos conceitos matemáticos inerentes. Outrossim, é destacada também uma abordagem matricial para gerar os números de Jacobsthal. No encerramento do trabalho é feita uma recapitulação do que foi exposto e salientada a importância dessa contribuição para a evolução matemática da sequência de Jacobsthal.

No artigo de Gananam e Anitha (2016), é proposta uma extensão aos inteiros para a sequência de Jacobsthal. Dessa forma, os autores utilizam a fórmula de Binet para discutir algumas propriedades matemáticas que possibilitam a obtenção desses termos. A partir dessas identidades, podemos utilizar para calcular as matrizes inversas associadas aos números de Jacobsthal. 
Os artigos apresentados anteriormente são voltados para um tratamento no âmbito da matemática pura. Assim, o caráter metodológico não é evidenciado. Porém, Alves (2017), apresenta em sua produção uma abordagem envolvendo uma preocupação metodológica. Dessa maneira, seu trabalho propõe uma Engenharia Didática para a s-sequência de Jacobsthal e para a s,t- Sequência de Jacobsthal, buscando realizar uma transposição didática do saberes científicos para o saber escolar . Destacamos nesse texto uma proximidade com o que tencionamos desenvolver, tendo em vista que propomos a utilização da Engenharia Didática em completude com a Teoria das Situações Didáticas.

Por fim, Aydin (2018) sugere um modelo de complexo para a sequência de Jacobsthal. Dessa forma, é feita uma extensão desses números para variável complexa. Não obstante, é evidenciada uma generalização para vetores, sob a perspectiva da sequência de Jacobsthal. Concluindo o texto, o autor apresenta diversas áreas do conhecimento como mecânica quântica, física quântica e etc.

Destarte, os trabalhos selecionados nos fornecem uma abordagem atualizada sobre o tema em questão e mostram que as pesquisas sobre sequência de Jacobsthal estão se difundindo em vários países. Isso mostra a importância de estudos desse tópico e sua inserção em aulas de História da Matemática.

No tópico a seguir destacamos o caráter de complementariedade entre a Engenharia Didática e a Teoria das Situações Didáticas, propondo situações de ensino nas quais serão elucidadas as possíveis atitudes dos estudantes em determinados tempos didáticos.

\section{Análises a priori e concepção e descrição das situações didáticas.}

Essa etapa é marcada pela construção dos princípios desejados pela aplicação da sequência didática. Nessa perspectiva, as variáveis da pesquisa necessitam estar bem definidas para que possam garantir o alcance dos objetivos esperados pela experimentação. A esse respeito, Artigue (1996) destaca dois tipos de variáveis: as macrodidáticas ou globais e as microdidáticas ou locais, conforme mencionadas anteriormente.

Destarte, na análise a priori devemos: descrever as escolhas das variáveis locais, relacionando-as sempre que possível com as globais; elaborar e desenvolver situações didáticas; investigar a importância da situação para o aluno e prever os comportamentos prováveis, mostrando de que modo a análise realizada pode controlar essas ações.

Assumimos posição em conformidade com PAIS (2015, p.102), quando afirma que: "Uma sequência didática é formada por certo número de aulas planejadas e analisadas previamente com a finalidade de observar situações de aprendizagem, envolvendo os 
conceitos previstos na pesquisa didática". Desse modo, reconhecendo o potencial de sequências didáticas e tendo em vista o que propomos, passaremos a discutir, com base nas fases da Teoria das Situações Didáticas, situações-problemas que foram pensadas para uma possível abordagem em sala de aula.

Situação Problema 1: Conforme mencionado anteriormente, sabemos que a fórmula de Binet empregada para números naturais é dada por $J_{n}=\frac{\left(2^{n}-(-1)^{n}\right)}{3}$. A partir dessa relação, mostre que $J_{-n}=\frac{(-1)^{n+1}}{2^{n}} \cdot J_{n}$, para todo 'n' inteiro.

Situação de Ação: Segundo Pais (2015): “uma situação de ação é aquela em que o aluno realiza procedimentos mais imediatos para resolução de um problema, resultando na produção de um conhecimento de natureza mais experimental e intuitiva do que teórica". Assim, o professor deve estimular a manipulação dos casos particulares. De maneira preliminar, o aluno pode confrontar as relações $J_{n}=\frac{\left(2^{n}-(-1)^{n}\right)}{3}$ e $J_{-n}=\frac{(-1)^{n+1}}{2^{n}} \cdot J_{n}$ procurando compreender e estabelecer familiaridades entre as mesmas.

Situação de Formulação: A situação de formulação é marcada pelas interações existentes em determinada atividade proposta. Tal situação é caracterizada pela troca de saberes entre alunos. Essa transferência de informações pode ser expressa, por meio de mensagens escritas ou orais. Além disso, a linguagem utilizada pelo aluno nessa fase pode ser a linguagem natural ou mesmo matemática. (ALMOULOUD, 2007). Desse modo, alguns casos particulares propostos em Gnanam \& Anitha (2016) podem ser motivados, a saber:

$J_{-1}=\frac{(-1)^{1+1}}{2^{1}} \cdot J_{1}=\frac{1}{2}, J_{-2}=\frac{(-1)^{2+1}}{2^{2}} \cdot J_{2}=-\frac{1}{4}, J_{-3}=\frac{(-1)^{3+1}}{2^{3}} \cdot J_{3}=\frac{3}{8}$. Vale mencionar que a posição assumida pelo professor, condutor do processo, é de extrema importância. Tendo em vista que o mesmo atuará como estimulador dos casos particulares descritos anteriormente. Por último, o docente poderá estimular o estudante a levantar a seguinte conjectura $J_{n}=\frac{\left(2^{n}-(-1)^{n}\right)}{3}$.

Situação de Validação: Nessa fase, o aprendiz tenta convencer um ou vários interlocutores da legitimidade das asserções que são feitas. Assim, conforme Pais (2015), esse tipo de situação 


\section{\#tear}

relaciona-se ao plano argumentativo racional e preocupa-se com a questão da veracidade do conhecimento.

Desse modo, utilizando a fórmula de Binet, temos a seguinte dedução:

$$
\begin{aligned}
& J_{-n}=\frac{\alpha^{-n}-\beta^{-n}}{3}=\frac{\frac{1}{\alpha^{n}}-\frac{1}{\beta^{n}}}{3}=\frac{\frac{1}{\alpha^{n}}}{3}-\frac{\frac{1}{\beta^{n}}}{3}=\frac{1}{3 \alpha^{n}}-\frac{1}{3 \beta^{n}}=\frac{\beta^{n}-\alpha^{n}}{3 \alpha^{n} \beta^{n}}=\frac{\left(\beta^{n}-\alpha^{n}\right)}{3(\alpha \beta)^{n}}=\frac{(-1)\left(\alpha^{n}-\beta^{n}\right)}{3(-2)^{n}}= \\
& =\frac{(-1)}{(-2)^{n}} \cdot \frac{\left(\alpha^{n}-\beta^{n}\right)}{3}=\frac{(-1)}{(-1)^{n} \cdot(2)^{n}} \cdot \frac{\left((2)^{n}-(-1)^{n}\right)}{3}=\frac{(-1)^{n+1}}{(2)^{n}} \cdot J_{n} .
\end{aligned}
$$

No fim, o aluno deve observar que os testes realizados para alguns valores de n, resultam na sequência de Jacobsthal como forma de extensão aos índices inteiros.

Situação de Institucionalização: No último estágio espera-se que o aprendiz ou grupo de aprendizes adote o significado de um saber que foi elaborado por ele mesmo ao longo das fases anteriores. Segundo Almouloud (2007, p.40), nessa situação "o professor fixa convencionalmente e explicitamente o estatuto cognitivo do saber". Dessa forma, o professor retorna as discussões feitas nas etapas anteriores, evidenciando a possibilidade de obtenção da fórmula de Binet, também no campo dos inteiros. O professor poderá ainda salientar que as relações $J_{-n} \neq-J_{n}$.

Situação Didática II: Considerando a relação apresentada na situação anterior e recordando as matrizes dadas anteriormente, as quais $F=\left[\begin{array}{ll}1 & 2 \\ 1 & 0\end{array}\right]$ e $F^{n}=\left[\begin{array}{cc}J_{n+1} & 2 J_{n} \\ J_{n} & 2 J_{n-1}\end{array}\right]$. Mostrar que para algum ' $\mathrm{n}$ ' inteiro

(i) $F^{-n}=\left[\begin{array}{ll}\frac{(-1)^{n}}{2^{n-1}} \cdot J_{n-1} & 2 \cdot \frac{(-1)^{n+1}}{2^{n}} \cdot J_{n} \\ \frac{(-1)^{n+1}}{2^{n}} \cdot J_{n} & 2 \cdot \frac{(-1)^{n+2}}{2^{n+1}} \cdot J_{n+1}\end{array}\right]=\left[\begin{array}{cc}J_{-(n-1)} & 2 J_{-n} \\ J_{-n} & 2 J_{-(n+1)}\end{array}\right] \mathrm{e}$

(ii) $J_{-(n-1)} J_{-(n+1)}-J_{-n}^{2}=(-1)^{n} \cdot 2^{-n-1}$

Situação de ação: Neste momento os alunos serão estimulados a observar com relação ao item (i) que a matriz $F^{-n}$ nada mais é que a matriz inversa da matriz $F^{n}$. Além disso, sobre o item (ii) os alunos poderão confrontar as relações $J_{n-1} J_{n+1}-J_{n}^{2}=(-1)^{n-1} \cdot 2^{n-1}$ e $J_{-(n-1)} J_{-(n+1)}-J_{-n}^{2}=(-1)^{n} \cdot 2^{-n-1}$ que são obtidas por meio da identidade de Cassini.

Situação de Formulação: Nesta ocasião, em relação ao item (i) os alunos serão motivados a analisar alguns casos particulares tais quais: 


$$
\begin{aligned}
& F^{-1}=\left[\begin{array}{cc}
0 & 1 \\
\frac{1}{2} & -\frac{1}{2}
\end{array}\right], F^{-2}=\left[\begin{array}{cc}
\frac{1}{2} & -\frac{1}{2} \\
-\frac{1}{4} & \frac{3}{4}
\end{array}\right]=\left[\begin{array}{cc}
\frac{(-1)^{2}}{2^{2-1}} \cdot 1 & 2 \cdot \frac{(-1)^{2+1}}{2^{2}} \cdot 1 \\
\frac{(-1)^{2+1}}{2^{2}} \cdot 1 & 2 \cdot \frac{(-1)^{2+2}}{2^{2+1}} \cdot 3
\end{array}\right], \\
& F^{-3}=\left[\begin{array}{cc}
-\frac{1}{4} & \frac{3}{4} \\
\frac{3}{8} & -\frac{5}{8}
\end{array}\right]=\left[\begin{array}{ll}
\frac{(-1)^{3}}{2^{3-1}} \cdot 1 & 2 \cdot \frac{(-1)^{3+1}}{2^{3}} \cdot 3 \\
\frac{(-1)^{3+1}}{2^{3}} \cdot 3 & 2 \cdot \frac{(-1)^{3+2}}{2^{3+1}} \cdot 5
\end{array}\right]
\end{aligned}
$$

Assim, o professor deve fomentar nos aprendizes que os mesmos observem que as matrizes $F^{-1}, F^{-2}, F^{-3}$ e $F^{-n}$, são respectivamente, as inversas das matrizes $F, F^{2}, F^{3}$ e $F^{n}$. Assim, a $\begin{array}{llll}\text { conjectura a seguir } & \text { sedé }\end{array}$ (i) $F^{-n}=\left[\begin{array}{ll}\frac{(-1)^{n}}{2^{n-1}} \cdot J_{n-1} & 2 \cdot \frac{(-1)^{n+1}}{2^{2}} \cdot J_{n} \\ \frac{(-1)^{n+1}}{2^{n}} \cdot J_{n} & 2 \cdot \frac{(-1)^{n+2}}{2^{n+1}} \cdot J_{n+1}\end{array}\right]=\left[\begin{array}{cc}J_{-(n-1)} & 2 J_{-n} \\ J_{-n} & 2 J_{-(n+1)}\end{array}\right]$

À respeito do item (ii) o aluno será provocado a calcular o determinante da matriz $F^{-1}$. Assim, $\operatorname{det}\left(F^{-1}\right)=\left(-\frac{1}{2}\right)$. Na fase subsequente empregaremos a indução matemática para provar o que foi conjecturado anteriormente.

Situação de Validação: Como mencionado anteriormente o aprendiz tenta convencer um ou vários interlocutores da legitimidade das asserções que são feitas. Assim, é necessário que os mesmos desenvolvam provas e demonstrações para assegurar a validade de suas descobertas. Desse modo, por indução obtemos o desejado para o item (i):

$$
\begin{aligned}
& F^{-n-1}=F^{-n} \cdot F^{-1}=F^{-n}=\left[\begin{array}{ll}
\frac{(-1)^{n}}{2^{n-1}} \cdot J_{n-1} & 2 \cdot \frac{(-1)^{n+1}}{2^{2}} \cdot J_{n} \\
\frac{(-1)^{n+1}}{2^{n}} \cdot J_{n} & 2 \cdot \frac{(-1)^{n+2}}{2^{n+1}} \cdot J_{n+1}
\end{array}\right] \cdot\left[\begin{array}{cc}
0 & 1 \\
\frac{1}{2} & -\frac{1}{2}
\end{array}\right]= \\
& =\left[\begin{array}{ll}
\frac{(-1)^{n+1}}{2^{n}} \cdot J_{n} & \frac{(-1)^{n+2}}{2^{n-1}} \cdot J_{n-1}+\frac{(-1)^{n+2}}{2^{n}} \cdot J_{n} \\
\frac{(-1)^{n+2}}{2^{n+1}} \cdot J_{n+1} & \frac{(-1)^{n+1}}{2^{n}} \cdot J_{n}+\frac{(-1)^{n+1}}{2^{n+1}} \cdot J_{n+1}
\end{array}\right]=\left[\begin{array}{ll}
\frac{(-1)^{n+1}}{2^{n}} \cdot J_{n} & \frac{(-1)^{n+2}}{2^{n}} \cdot\left(2 J_{n-1}+J_{n}\right) \\
\frac{(-1)^{n+2}}{2^{n+1}} \cdot J_{n+1} & \frac{(-1)^{n+3}}{2^{n+1}} \cdot\left(2 J_{n}+J_{n-1}\right)
\end{array}\right]= \\
& =\left[\begin{array}{ll}
\frac{(-1)^{n+1}}{2^{n}} \cdot J_{n} & 2 \cdot \frac{(-1)^{n+2}}{2^{n+1}} \cdot\left(J_{n+1}\right) \\
\frac{(-1)^{n+2}}{2^{n+1}} \cdot J_{n+1} & 2 \cdot \frac{(-1)^{n+3}}{2^{n+2}} \cdot\left(J_{n+2}\right)
\end{array}\right] \text { (c.q.d) }
\end{aligned}
$$




\section{\#tear}

Com relação ao item (ii), utilizaremos procedimentos análogos ao caso da extensão de Cassini para números naturais. Desse modo, obtemos:

$$
\left.\operatorname{det} F^{-n}=\operatorname{det}\left[\begin{array}{cc}
J_{-(n-1)} & 2 J_{-n} \\
J_{-n} & 2 J_{-(n+1)}
\end{array}\right]=2\left(J_{-(n-1)} J_{-(n+1)}-J_{-n}^{2}\right)=\left(-\frac{1}{2}\right)^{n}=(-1)^{n} \cdot 2^{-n-1} \text { (c.q.d }\right)
$$

Situação de Institucionalização: Por fim, a mediação do professor será finalizada com a apresentação das principais propriedades formais que asseguram a consistência das operações e manipulações realizadas nas fases anteriores (ALVES, 2016). Dessa forma, ao retornarmos as fases anteriores percebemos a possibilidade da extensão aos inteiros para outros conteúdos matemáticos, tais como matrizes e o estudo de determinantes explorados por meio da fórmula de Cassini. Na figura 3 apresentamos ao leitor um movimento dialético necessário para subsidiar a atividade e mediação do professor de Matemática.

Figura 3 - Margolinas (2015) descreve a dialética entre conhecimento/saber e seu importante papel para a Didática da Matemática e função no ensino.

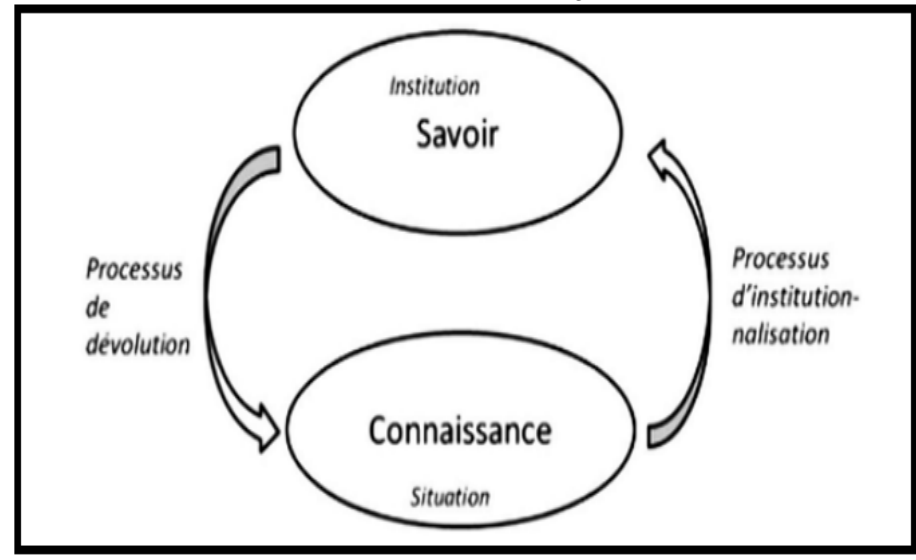

Fonte: Margolinas (2015)

Assim, observamos o potencial da Teoria das Situações Didática, no que diz respeito à possibilidade de assinalar e descrever estágios específicos envolvendo as relações existentes entre estudante, professor e saber. A boa consecução dessas relações são irremediavelmente determinantes para a construção de um conhecimento matemático.

Urge o entendimento, por parte do professor de Matemática, como um autêntico elaborador e um construtor de situações problema, cujo conhecimento matemático adquire/assume um caráter duplo. Como observamos no excerto anterior, na situação da ação, o conhecimento funciona e desempenha o papel preliminar como um instrumento, um constructo técnico com o fim na resolução de problemas específicos. Mas, no caso de uma situação de validação, por exemplo, o próprio status do saber é objetivado. Entra em cena, pois, o interesse pelo próprio saber matemático empregado/mobilizado em situação, 


\section{\#tear}

perspectivado agora como um objeto teórico-conceitual, constituinte de um conhecimento científico mais amplo. Todavia, o exame imprescindivel de análise e apreciação do professor de Matemática necessita dessa flexibilidade de mudança de perspectiva e, desse modo, provocar as modificações esperadas nos estudantes e potencializar suas escolhas.

\section{Conclusão}

Destarte, neste trabalho apresentamos considerações iniciais sobre a sequência de Jacobsthal, apresentando a fórmula de Binet como modelo de generalização desse conceito e, além disso, observamos a extensão dessa fórmula a outros conceitos matemáticos, estabelecendo uma inter-relação entre conteúdos.

Discorremos também sobre uma Engenharia Didática Clássica, pautada na experimentação, tendo como base a Teoria das Situações Didáticas, que se configura como uma teoria específica e intimamente relacionada aos conceitos matemáticos. O emprego da ED busca a obtenção, acúmulo, constatação e determinação de conhecimentos técnicos, didáticos e metodológicos sobre uma prática controlada de transmissão e transposição didática, relativamente a determinado conteúdo matemático (ALVES, 2017).

Assim, as fases de análises preliminares e análises a priori da ED, possibilitaram a demarcação de aspectos de ordem epistemológica, didática e cognitiva, bem como a análise de variáveis didáticas para a elaboração de situações de ensino, que propõem apresentar propostas de problemas que podem ser replicadas em sala de aula (ALVES; ALVES DIAS, 2017).

Por fim, esperamos que o percurso metodológico abordado neste trabalho funcione como referência para organizar e sistematizar futuras pesquisas no âmbito do ensino de matemática, fundamentadas nos aspectos da Engenharia Didática em complementariedade com a Teoria das Situações Didáticas.

\section{DIDACTIC ENGINEERING AS A METHODOLOGICAL INSTRUMENT IN THE STUDY AND TEACHING OF THE JACOBSTHAL SEQUENCE}

Abstract: The work on screen is intended to discuss some models of generalization extension Jacobsthal Sequence. Thus, in accordance with the guidelines of the Didactic Engineering - DE as research methodology and in complementarity with the Theory of Educational Situations - TES that allows a detailed analysis of the phenomena related to the teaching and learning of Jacobsthal Sequence, we will describe the main elements that constitute the two initial phases of an DE, namely preliminary analysis and a priori analysis, 
highlighting the conception of didactic situations and the possible behaviors and resolution of the students before the two problem situations, organized for purposes of experimentation.

Keywords: Didactic Engineering. Theory of Educational Situations. sequence of Jacobsthal.

\section{Referências}

ALMOULOUD, S. Fundamentos da Didática da Matemática. Paraná: Universidade Federal do Paraná, 2007.

ALMOULOUD, Saddo Ag; SILVA, Maria José Ferreira da. Engenharia didática: evolução e diversidadeDidactic engineering. Revemat: revista eletrônica de educação matemática, [s.1.], v. 7, n. 2, p.22-52, 13 dez. 2012.

ALVES, Francisco Regis Vieira. Engenharia Didática para a generalização da sequência de Fibonacci: uma experiência num curso de licenciatura. Revista de Educação Matemática e Pesquisa, São Paulo. v.18, n.1, p.61-93, 2016.

ALVES, Francisco Regis Vieira. Engenharia Didática para a s-Sequência Generalizada de Jacobsthal e a (s,t)-Sequência Generalizada de Jacobsthal: análises preliminares e a priori. Revista Unión, São Paulo, v. 12, n. 41, p.83-106, dez. 2017.

ALVES, Francisco Regis Vieira. Engenharia Didática de Formação (EDF): sobre o ensino dos Números (Generalizados) de Catalan (NGC) Didactical Engineering. Educação Matemática Pesquisa: Revista do Programa de Estudos Pós-Graduados em Educação Matemática, [s.1.], v. 20, n. 2, p.47-83, 3 out. 2018.

ALVES, Francisco Regis Vieira.; ALVES DIAS, Marlene. Formação de Professores de Matemática: um contributo da Engenharia Didática. REVEMAT, v. 12, nº 2, 192 - 209. Out. 2017.

ARTIGUE, Michelle. Ingenieria Didática. Artigue, Michelle ; Douady, Régine ; Moreno, Luis \& Gomez, Pedro. In: Ingeniéria didatica en Educacion Matemática. Bogotá : Grupo Editorial Iberoamericano, pp. 33-61, 1995b. Disponível

em:〈http://funes.uniandes.edu.co/676/1/Artigue1995Ingenieria.pdf.> . Acesso em: 10 abr. 2018.

AYDIN, Fügen Torunbalc1. On generalizations of the Jacobsthal sequence. Notes On Number Theory And Discrete Mathematics, [s.1.], v. 24, n. 1, p.120-135, mar. 2018. 24.1.120-135.

BACHERLARD, Gaston. A formação do espirito científico: contribuição para uma psicanálise do conhecimento. Tradução: Estela dos Santos Abreu. Rio de Janeiro, RJ: Contra ponto, 1996.

BROUSSEAU, G. Les Obstacles 'epist'emologicques et les problems en mathematiques. Comptes rendus de la XXVIIIe rencontre organisée par la Commission Internationale pour l'Etude et l', Louvain-la-neuve, pp.101-117, 1976. 
CAMPOS, H. et al. On some identities of k-Jacobsthal-Lucas numbers. International Journal Of Mathematical Analysis, [s.1.], v. 8, p.489-494, 2014

CATARINO, Paula et al. New families of Jacobsthal and Jacobsthal-Lucas numbers. Journal Of Algebra And Discrete Mathematics, [s.1.], v. 20, n. 1, p.40-54, jan. 2015.

COOK, Charles K; BACON, Michael R. Some identities for Jacobsthal and Jacobsthal-Lucas numbers satisfying higher order recurrence relations, Ann. Math. Inform, 41, 2013, 27-39.

CRAVEIRO, Irene Magalhães. Extensões e interpretações combinatórias para os números de Fibonacci, Pell e Jacobsthal. 2004. 108 p. Tese (doutorado) - Universidade Estadual de Campinas, Instituto de Matematica, Estatistica e Computação Cientifica, Campinas, SP. Disponível em: <http://repositorio.unicamp.br/jspui/handle/REPOSIP/307506>. Acesso em: 28 mar. 2018.

FERREIRA, Norma Sandra de Almeida. As pesquisas denominadas "estado da arte". Revista Educação \& Sociedade, Campinas, n. 79, p. 257-272, Ago, 2002

GNANAM, A.; ANITHA, B. (2016). Negative Jacobsthal Numbers. International Journal of Science, Engineering and Technology Research (IJSETR). v. 5, n.3, 663 - 665

HORADAM, Asd Fassd. Jacobsthal representation numbers. The Fibonacci Quarterly. Austrália, p. 40-55. fev. 1996.

JACOBSTHAL, E. (1919 - 1920). Fibonaccische Polynome und Kreisteilungsgleichungen. Berlinger Mathematische Gesellschaft. Sitzungsberichte. v. 13, 43 - 57.

KOKEN, Fikri; BOZKURT, Durmus. On the Jacobsthal numbrs by matrix methods. International Journal of Contemporary Mathematics. v.3, n. 13, 605-614, 2008. Disponível em:< http://www.m-hikari.com/ijcms-password2008/13-16-2008/kokenIJCMS1316-2008.pdf.>. Acesso em 05 mai. 2018.

KOSHY. T. Fibonacci and Lucas Numbers And Applications. New York: John Willey and Sons, 2011.

MARGOLINAS, C. Situations, savoirs et connaissances... comme lieux de rencontre? Formation et pratiques d'enseignement en questions. v. 1, n. 19, 31-39, 2015.

SILVA, Kênia Cristina Pereira. Sobre questões de combinatória envolvendo os números de Fibonacci, Pell e Jacobsthal. 2014. 151 p. Tese (doutorado) - Universidade Estadual de Campinas, Instituto de Matemática Estatística e Computação Científica, Campinas, SP. Disponível em: <http://repositorio.unicamp.br/jspui/handle/REPOSIP/307508>. Acesso em: 28 mar. 2018.

SPREAFICO, Elen Viviani Pereira. Novas identidades envolvendo os números de Fibonacci, Lucas e Jacobsthal via ladrilhamentos. 2014. 132 p. Tese (Doutorado) Universidade Estadual de Campinas, Instituto de Matemática Estatística e Computação Científica, Campinas, SP. Disponível em: <http://repositorio.unicamp.br/jspui/handle/REPOSIP/307509 >. Acesso em: 28 mar. 2018. 
PAIS, Luiz Carlos. Didática da Matemática: Uma análise da influência francesa. Belo Horizonte: Autêntica, 2015.

POMMER, W. M. A Engenharia Didática em sala de aula: Elementos básicos e uma ilustração envolvendo as Equações Diofantinas Lineares. São Paulo: [s.n.], 2013. 